\title{
The preclinical phase of PsA: a challenge for the epidemiologist
}

\section{Alexis Ogdie}

Psoriatic arthritis (PsA) is a chronic inflammatory arthritis and, like many chronic diseases, often has an insidious onset. The disease likely begins well before patients first present to a rheumatologist and even before they first have symptoms. Several studies have confirmed the presence of subclinical joint and entheseal inflammation in patients with psoriasis. ${ }^{1-5}$ However, relatively little is known about the preclinical phase of PsA. The 'preclinical phase' is emerging as an important issue in many rheumatic diseases and is an important area of ongoing research. A preclinical phase in rheumatoid arthritis (RA) has been fairly well described. Current research suggests that, among predetermined or genetically susceptible individuals, an inciting agent (eg, smoking) ignites asymptomatic inflammation. This is followed by asymptomatic synovitis, development of symptoms, a transition to clinically apparent RA and subsequent diagnosis, and then a chronic inflammatory phase. ${ }^{6-8}$ The preclinical phase of RA is supported by studies identifying the presence of autoantibodies $\geq 10$ years prior to presentation of clinically apparent disease in RA. ${ }^{9}$

In this issue of the Annals of Rheumatic Disease, Kristensen et al ${ }^{10}$ provide a population-based description of the period leading up to the diagnosis of PsA, potentially the 'preclinical phase' of PsA in their paper 'Societal costs and patients' experience of health inequities before and after diagnosis of psoriatic arthritis'. In this paper, we see that healthcare costs begin to rise approximately 5 years prior to the diagnosis of PsA, peak around the time of diagnosis, improve slightly (likely from institution of therapy and appropriate care) but then remain high (figure 1). Additionally, the investigators demonstrate an elevated prevalence of comorbidities compared with the general population both in the three years before and three years after diagnosis of PsA.

The findings in this study highlight the interesting and often complex issues encountered in designing and interpreting

Correspondence to Dr Alexis Ogdie, Division of Rheumatology, Center for Clinical Epidemiology and Biostatistics. Perelman School of Medicine at the University of Pennsylvania, White Building, Room 5023, 3400 Spruce St., Philadelphia, PA 19104, USA; alexis. ogdie@uphs.upenn.edu epidemiological studies of PsA. In particular, the elevated prevalence of comorbidities before PsA diagnosis has implications for studies examining comorbidities linked to PsA and studies aiming to identify risk factors for PsA. For both study designs, the critical issue is the definition of when PsA begins and how to manage this potential preclinical or prediagnosis phase of PsA.

\section{DISENTANGLING 'PRECLINICAL' PSA FROM PSORIASIS AND/OR DELAYED DIAGNOSIS: THE ULTIMATE CHALLENGE}

Studying the preclinical phase of rheumatic disease from a population-based perspective is challenging, and this is particularly true for PsA because it is complicated by the coexistence of psoriasis, another disorder associated with systemic inflammation. It is possible that during a subclinical phase of the disease asymptomatic inflammation leads to development of comorbidities and socioeconomic disability, as seen in Kristensen et al. Inflammatory states may increase fatigue and malaise, and subsequently, patients miss work, they see the doctor and get more tests and more problems are identified (and thus, this period prior to diagnosis is complicated by potential observation and/or ascertainment bias). However, one wonders whether comorbidities preceding PsA could be in fact attributed to psoriasis. The majority of patients with PsA have psoriasis and the average duration of psoriasis at the time of PsA diagnosis is approximately $7-10$ years. ${ }^{11}$ Even mild psoriasis is associated with comorbidities including cardiovascular disease. ${ }^{12}{ }^{13}$ Alternatively, these findings may be explained by delayed diagnosis of clinically apparent (rather than clinically asymptomatic) PsA, as reported in previous studies. ${ }^{1415}$ Regardless of the explanation of the findings in Kristensen et al, the interval from start of asymptomatic inflammation to diagnosis is particularly important for the design and interpretation of PsA epidemiology studies.

DEFINING RISK FACTORS FOR THE DEVELOPMENT OF PSA: MANAGING THE TROUBLING PRECLINICAL PHASE The most challenging aspect of the preclinical phase of PsA is managing its potential existence in studies of risk factors for PsA. In some ways, PsA is the perfect disease for which to identify risk factors. There is a known 'risk pool' (patients with psoriasis) that may develop the disease. And theoretically, if we could identify risk factors for the disease, we could either diagnose the disease earlier (and improve long-term outcomes) or potentially even mitigate the risk for developing the disease by removing the risk factor (eg, smoking cessation). We have previously discussed more general methodological considerations in risk factor studies in PsA. ${ }^{16}$ However, this study raises a new concern. The risk assessment window typically used in risk factor studies (shown in figure 1) may be biased: if in the 3-5 years preceding a PsA diagnosis the patient actually has preclinical PsA, we may identify 'risk factors' that are part of the disease rather than true aetiological or causal factors. Casecontrol studies (which start at diagnosis and look back for exposures) and studies using time-updated exposures/covariates would be particularly at risk for this bias. (An aside: Time-updated exposures are used to acknowledge and account for the fact that people may change exposure status over time during long observation periods. Cox proportional hazards models can account for these changes over time. Time-updated exposures also can be problematic for other reasons if you suspect that the value of the exposure at one time point influences its value at a subsequent time point. We'll set that aside for now. ${ }^{17}$ ) Let's use socioeconomic status (SES) as an example. Kristensen et al found that SES effectively worsens in the 3-5 years prior to PsA diagnosis. If we use a case-control design (or a cohort study using SES as a time-updated risk factor), we will come to the conclusion that lower SES is a risk factor for PsA because, as long as the data are available, the risk factor will be assessed closest to diagnosis. However, it may be that lower SES is instead the result of preclinical PsA. This is problematic if we are aiming to establish causality. Potential ways to address this problem include (a) cohort study designs in which risk factors are identified at baseline only (eg, at psoriasis diagnosis or start of follow-up) or (b) sensitivity analyses restricting the window during which risk factors are assessed (eg, closing the risk factor assessment window 3-5 years prior to diagnosis). On the other hand, if we are looking for factors that may signal onset of clinical symptoms and we aim to detect these patients earlier, then potentially the window should be limited to the 


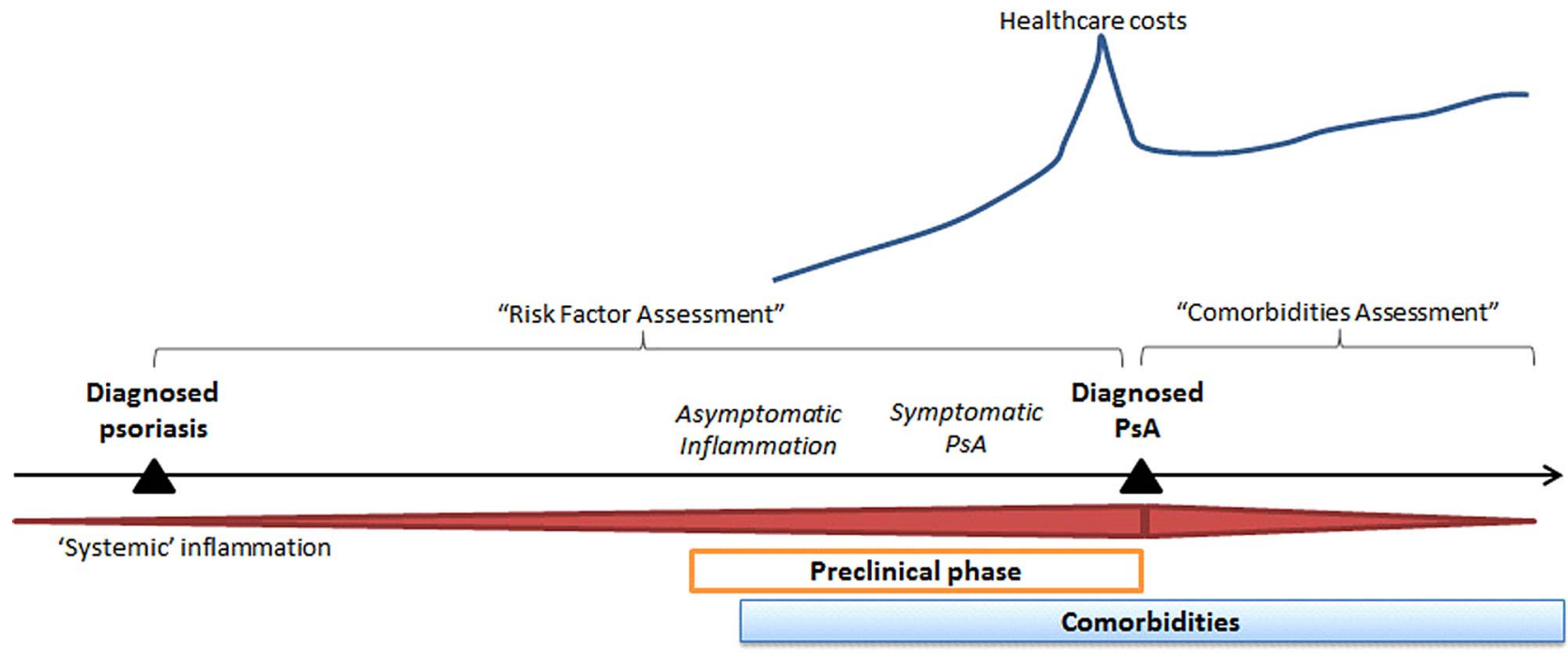

Figure 1 A preclinical phase of psoriatic arthritis (PsA) likely includes an asymptomatic inflammatory phase followed by development of symptoms and ultimately diagnosis. Kristensen et al demonstrated increasing comorbidities and societal economic costs in the 3 and 5 years prior to diagnosis, respectively. Disentangling the 'preclinical phase' of PsA from psoriasis is challenging. Furthermore, this preclinical phase makes assessment of comorbidities and risk factors challenging by impacting the interval over which risk factors and comorbidities may be assessed.

3-5 years prior to diagnosis. This issue reinforces the importance of having a prespecified question (eg, are we seeking potentially causal/aetiological factors or preclinical factors for earlier disease identification?) and designing the study accordingly.

Now, to flip the coin: maybe comorbidities and SES are truly causal risk factors for the development of PsA among patients with psoriasis and this study provides evidence for these risk factors (although a comparison cohort of patients with psoriasis who didn't develop PsA is not included in the study). Obesity and hyperlipidaemia have been identified as risk factors for psoriasis and PsA. ${ }^{18-21}$ Biological plausibility exists: comorbidities may be associated with inflammation, an altered endocrine state or mechanical/ sheer forces (in the case of obesity) ${ }^{22}-$ so, these are possible legitimate triggers for disease onset. In this case, these factors should theoretically be positively associated regardless of the window during which they are assessed (although with potentially differing effect sizes). Thus, investigators should consider assessing both windows in separate analyses to confirm the association.

\section{IMPLICATIONS FOR EXAMINING RISK OF DEVELOPMENT OF COMORBIDITIES AMONG PATIENTS WITH PSA}

This preclinical phase also makes understanding relationships between the disease and comorbid conditions difficult. In assessing the risk for comorbidities in patients with PsA, we often 'start' follow-up time at the diagnosis of PsA ('comorbidities assessment' window in figure 1). We do this in order to examine whether comorbidities can be attributed to the disease rather than pre-existing conditions. Additionally, as incidence is calculated as the number of new cases in the population at risk for the disease, patients with pre-existing comorbidities are not included in this calculation. The study by Kristensen et al suggests that if we start the follow-up time at the diagnosis of PsA, we may be falsely assuming a lower incidence of comorbidities by excluding the initial cases when, in fact, these initial cases may actually have been caused by the preclinical phase of the disease. This concept is known as 'depletion of the susceptibles'-the patients most at risk for developing these comorbidities may be excluded (depleted) from the study because they have already developed the comorbidity before study follow-up begins. Following only patients without the disease at baseline may lower the HR relating the risk of an incident comorbidity in patients with PsA compared with the general population. In a recent study examining the risk of fracture among patients with PsA, we managed this potential bias by including a sensitivity analysis in which patients with a fracture prior to start date were included and we adjusted for previous fracture to determine whether it substantially changed the HR. ${ }^{23}$ Investigators could also consider moving back the start date by 3 years, for example. Standard analyses are still informative and do appropriately answer the clinically relevant question, 'For this patient with a diagnosis of PsA, what is the likelihood of this patient developing a particular comorbidity?' The bigger problem is attribution; in calculating attributable risk of fracture related to PsA, we will have missed some of the new fractures that could in fact be related to the disease (in the preclinical phase) but occurred prior to formal diagnosis.

\section{FUTURE CONSIDERATIONS}

In summary, the paper by Kristensen et al raises many important questions about the years prior to PsA diagnosis and how to best study both the risk factors for PsA and the long-term outcomes related to PsA. Population-based studies of PsA have several strengths; studies addressing risk factors for the development of PsA and long-term outcomes would not be possible without large generalisable populations of patients with physician-diagnosed PsA, physician recording of important covariates (diagnoses, lifestyle habits and medications) and several years of follow-up. However, care should be taken to interpret findings from epidemiology studies of PsA in light of this potential preclinical phase, and sensitivity analyses to address this window should be considered. In the bigger picture, this study by Kristensen et al suggests that this disease, once thought to be a relatively benign condition, has costly outcomes. It also suggests, however, that there may be a crucial period during which we can identify and intervene upon patients with early PsA in order to improve outcomes. $^{24}$ 
Acknowledgements The author thanks Joshua $F$ Baker, MD MSCE, and Michael George, MD MSCE, for helpful discussions.

Funding National Institute of Arthritis and Musculoskeletal and Skin Diseases (grant no. K23 AR063764) and the Rheumatology Research Foundation.

Competing interests $\mathrm{AO}$ has received support from an investigator-initiated research grant from Pfizer and has consulted for Novartis and Pfizer and has received payment for continuing medical education work related to psoriatic arthritis.

Provenance and peer review Commissioned; externally peer reviewed.

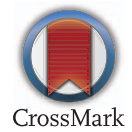

To cite Ogdie A. Ann Rheum Dis 2017;76:1481-1483.

Received 6 February 2017

Accepted 19 February 2017

Published Online First 8 March 2017

\section{C) Linked}

- http://dx.doi.org/10.1136/annrheumdis-2016210579

Ann Rheum Dis 2017:76:1481-1483.

doi:10.1136/annrheumdis-2017-211109

\section{REFERENCES}

1 Ash ZR, Tinazzi I, Gallego CC, et al. Psoriasis patients with nail disease have a greater magnitude of underlying systemic subclinical enthesopathy than those with normal nails. Ann Rheum Dis 2012;71:553-6.

2 Freeston JE, Coates LC, Nam JL, et al. Is there subclinical synovitis in early psoriatic arthritis?
A clinical comparison with gray-scale and power Doppler ultrasound. Arthritis Care Res (Hoboken) 2014;66:432-9.

3 Offidani A, Cellini A, Valeri G, et al. Subclinical joint involvement in psoriasis: magnetic resonance imaging and X-ray findings. Acta Derm Venereol 1998;78:463-5.

4 Faustini F, Simon D, Oliveira I, et al. Subclinical joint inflammation in patients with psoriasis without concomitant psoriatic arthritis: a cross-sectiona and longitudinal analysis. Ann Rheum Dis 2016;75: 2068-74.

5 McGonagle D, Ash Z, Dickie L, et al. The early phase of psoriatic arthritis. Ann Rheum Dis 2011;70(Suppl 1):i71-6.

6 Arend WP, Firestein GS. Pre-rheumatoid arthritis: predisposition and transition to clinical synovitis. Nat Rev Rheumatol 2012;8:573-86.

7 Deane KD. Learning about the natural history of rheumatoid arthritis development through prospective study of subjects at high risk of rheumatoid arthritis-related autoimmunity. Arthritis Rheum 2012;64:1708-12.

8 Mankia K, Emery P. Preclinical rheumatoid arthritis: progress toward prevention. Arthritis Rheum 2016:68:779-88.

9 Klareskog L, Rönnelid J, Lundberg K, et al. Immunity to citrullinated proteins in rheumatoid arthritis. Annu Rev Immunol 2008;26:651-75.

10 Kristensen LE, Jørgensen TS, Christensen R, et al. Societal costs and patients' experience of health inequities before and after diagnosis of psoriatic arthritis: a Danish cohort study. Ann Rheum Dis 2017. doi:10.1136/annrheumdis-2016-210579 [Epub ahead of print: 30 Jan 2017].

11 Gladman DD, Antoni C, Mease P, et al. Psoriatic arthritis: epidemiology, clinical features, course and outcome. Ann Rheum Dis 2005;64(Suppl 2) ii14-17.

12 Yeung H, Takeshita J, Mehta NN, et al. Psoriasis severity and the prevalence of major medical comorbidity: a population-based study. JAMA Dermatol 2013;149:1173-9.

13 Ogdie A, Yu Y, Haynes K, et al. Risk of major cardiovascular events in patients with psoriatic arthritis, psoriasis and rheumatoid arthritis: a population-based cohort study. Ann Rheum Dis 2015;74:326-32.

14 Mease PJ, Gladman DD, Papp KA, et al. Prevalence of rheumatologist-diagnosed psoriatic arthritis in patients with psoriasis in European/North American dermatology clinics. J Am Acad Dermatol 2013;69:729-35.

15 Sørensen J, Hetland ML. Diagnostic delay in patients with rheumatoid arthritis, psoriatic arthritis and ankylosing spondylitis: results from the Danish nationwide DANBIO registry. Ann Rheum Dis 2015;74:e12.

16 Ogdie A, Gelfand JM. Clinical risk factors for the development of psoriatic arthritis among patients with psoriasis: a review of available evidence. Curr Rheumatol Rep 2015;17:64

17 Robins JM, Hernán MA, Brumback B. Marginal structural models and causal inference in epidemiology. Epidemiology 2000;11:550-60.

18 Wu S, Li WQ, Han J, et al. Hypercholesterolemia and risk of incident psoriasis and psoriatic arthritis in US women. Epidemiology 2014;66:304-10.

19 Li W, Han J, Qureshi AA. Obesity and risk of incident psoriatic arthritis in US women. Ann Rheum Dis 2012;71:1267-772.

20 Love TJ, Zhu Y, Zhang Y, et al. Obesity and the risk of psoriatic arthritis: a population-based study. Ann Rheum Dis 2012;71:1273-7.

21 Soltani-Arabshahi R, Wong B, Feng BJ, et al. Obesity in early adulthood as a risk factor for psoriatic arthritis. Arch Dermatol 2010;146:721-6.

22 Russolillo A, lervolino S, Peluso $R$, et al. Obesity and psoriatic arthritis: from pathogenesis to clinical outcome and management. Rheumatology (Oxford) 2013;52:62-7.

23 Ogdie A, Harter L, Shin D, et al. The risk of fracture among patients with psoriatic arthritis and psoriasis: a population-based study. Ann Rheum Dis 2017;76:882-5.

24 Tillett W, Jadon D, Shaddick G, et al. Smoking and delay to diagnosis are associated with poorer functional outcome in psoriatic arthritis. Ann Rheum Dis 2013;72:1358-61. 